\title{
Allocation and routing ambulances under uncertainty condition and risk for demands using the multi-stage hybrid robust model
}

\author{
Ardavan Babaei ${ }^{\mathrm{a}}$ and Kamran Shahanaghi ${ }^{\mathrm{a}^{*}}$
}

${ }^{a}$ Department of Industrial Engineering, Iran University of Science and Technology, Tehran, Iran

\begin{tabular}{l}
\hline C H R O N I C L E \\
\hline Article history: \\
Received July 2, 2016 \\
Received in revised format \\
September 10, 2016 \\
Accepted December 82016 \\
Available online \\
December 82016 \\
\hline Keywords: \\
Emergency station \\
Ambulance \\
Allocation-Routing \\
Uncertainty Condition \\
SA algorithm
\end{tabular}

\section{Introduction}

Occurrence of unpredicted incidents and diseases always impacts various parts of the world especially in the big cities. Usually, incidents and unexpected diseases need quick relief regarding the financial and therapeutic limitations. The process of planning, management and control of the flow of relief resources for the injured people and patients is called relief logistics. The relief logistics seeks to present the best possible relief services to the people in need of relief with the available resource (Overstreet, 2011). Disasters are characterized by their uncertain and unpredictable natures; and therefore, demand may change rapidly in such an environment (Swiss, 2015). Performance of emergency services is measured in terms of the response time and the total logistics cost (Rahmaniani \& Shafia, 2013). Iran is a western Asian country, located in the central Asian and Caucasus region, with an area of approximately $1,650,000 \mathrm{~km}^{2}$ and a population of more than 75 million people. According to the World Health Organization (WHO), cardiovascular diseases are the most prevalent causes of mortality in Iran

* Corresponding author

E-mail address: shahanaghi@iust.ac.ir (K. Shahanaghi)

C 2017 Growing Science Ltd. All rights reserved. doi: $10.5267 /$ j.uscm.2016.12.001 
(Bahadori et al., 2010). Unintentional accidents are the second cause of mortality. Iran with 28000 annual deaths has the highest mortality rate from road traffic accidents in the world. The road accidents rate in Iran is 20 times higher than the world average. According to the Iranian data one person loses his/her life every 19 minutes due to car accidents (Bahadori et al., 2010). Iran is also constantly exposed to natural disasters such as earthquakes and floods. This situation further emphasizes the importance of an integrated system of relief logistics in place throughout the nation, because such a system benefits not only individual people but also serves the best interests of the nation. The researches in the humanitarian logistics operation have different levels including one-level, two-level and multi-level (Balcik \& Beamon, 2008). The number of facilities in this category of problems include one-facility and multi-facilities (Jotshi, et al., 2009). The scale of transport fleet is investigated for one vehicle and several vehicles (Tzeng, et al., 2007).

Ratick et al. (2009) declared that the emergency request is stochastic and fuzzy in certain humanitarian logistics operation. Mete and Zabinsky (2007) showed that availability of facilities and paths could be studied by two criteria of certain and uncertain conditions. The limitation of facilities capacity in all types of models was active and sometimes passive. The emergency vehicles were considered under two types of with capacity and without capacity. The time window was considered in three categories of unlimited time, soft time window and hard time window (Özdamar et al., 2004). The humanitarian logistics model can perform the delivery operation, loading and simultaneous delivery and loading in the stations (Campbell et al., 2008). The type of path is defined under two types of open and closed (Jotshi, et al., 2009). The location of customers in the network needs routing of nodes or edges (Mete $\&$ Zabinsky, 2007). The type of objective functions is cost, humanitarian or the combination of the two which are single-objective, two or multiple objective (Mete \& Zabinsky, 2007). These models can be employed in crisis such as earthquake, flood and others (Özdamar, et al., 2004; Mete \& Zabinsky, 2007). Modeling of location problems of emergency facilities has been emerged in scientific discussions with the onset of World War II and the researchers have paid special attention to the research in this field. The 1970s can be considered as the commencement of modeling of emergency facilities location. The first model was used to minimize the response time instead of target envelopment function (Swoveland et al., 1973). Between 1970 and 1980, most of the models have been considered in certain form and uncertainty was less in the warmth of respect. The first stochastic model was proposed by Aly and White (1978) and in 1980s, most of the researchers considered the models to be stochastic and the parameters to be random. Meanwhile, the models with supportive cover (ReVelle, 1989) were more attractive. One of the most important stochastic models with envelopment target function which provides a field for the application of stochastic models was presented by Daskin (1983). Moreover, in this period, location allocation (Pirkul \& Schilling, 1988) models were considered. The first review model which categorized the emergency facilities models was presented by ReVelle (1989). Also, Matsutomi and Ishii (1992) utilized fuzzy concept in modeling and solving the emergency location problems for the first time. The first model with considering the reliability was presented by Ball and Lin (1993). From 1990 to 2000, the researchers developed the existing models by focusing on above models. From 2000 on, there was a great attention to the solution methods and the multiple objective models were more popular during these years. In 2004, the partial cover model was presented by Karasakal and Karasakal (2004) which solves the main problem of maximal covering location problem (MCLP) model. Furthermore, receiving a lot of attention to solution methods caused that heuristic and meta-heuristic approaches play a significant role in the papers published in recent years. Based on the purpose we should minimize the time of travel and the loading time with regards to the path and extent of traffic flow should be evaluated to introduce effective factors in the decisionmaking (Campos et al., 2012). In order to shorten the distance between demand and traveling time limitation and road damages, the third model is investigated (Shen et al., 2008) with regards to the fourth basis which is the number of cases that the request is not satisfied, the number of needed emergency units, the total number of covered requests, minimizing the risk and increasing the survival of people, decreasing the waiting time of injured people for help, the volume of traffic and capacity of havens based on the number of vehicle, request, the covered area, cost and time of travel has been 
created (Campos et al., 2012). Location and allocation in humanitarian logistics transportation aims to minimize the transportation time with regards to the servicing, emergency demands and budget and resource constraints to increase the lifespan of the patients (Edrissi et al., 2013). However, based on the distribution, humanitarian logistics models have objectives such as cost which includes minimizing traveling costs, distribution cost and distance which their feasible space is proportional to the extent of supply (Liberatore et al., 2014). On the other hand, the capacity of facilities is one of the main elements in these problems with regards to the demand satisfaction. Therefore, issues such as extent of stock, resource flow and the number of injuries falls into this category (Davis et al., 2013). Limited financial power for implementing and managing of the ambulance stations is the reason why planning is largely necessary in this field. In addition, because of uncertain conditions in ambulance demand and the traversing time of the ambulances through the path in the various times of the day, uncertain planning will yield more real applications. All in all, a notable missing of the aforementioned researches is the lack of considering the stability of the problem space initially. By obviating this gap, the decisions will be accompanied by desirable and stable results. On the other hand, the integrated modeling of locating the emergency stations, allocating the ambulance and routing can lead to the better and more precise results. Also considering queue system of type $\mathrm{M} / \mathrm{M} / \mathrm{m}$ at steady state is another innovation. Minimizing queue length leads to faster response in relief logistics. According to this requirements, a comprehensive algorithm for location of emergency centers, allocation and routing of ambulance under uncertain condition is studied using a SA algorithm to solve the problem. In turn, a hybrid robust multistep planning is used which relies on chance due to the interaction with the decision maker.

The organization of this paper is as follows: in Section 2, the proposed model is presented. In Section 3 , numerical analysis is provided to analyze the model. Finally, in Section 4 , the concluding remarks is presented and suggestions for further studies are recommended.

\section{Problem definition}

Ambulance planning is one of the most important issues in relief logistics which faces with several challenges that make the decision making more complex. The proposed solution algorithm which is used in this paper is represented in Fig. 1. The steps of algorithm are explained in following subsections.

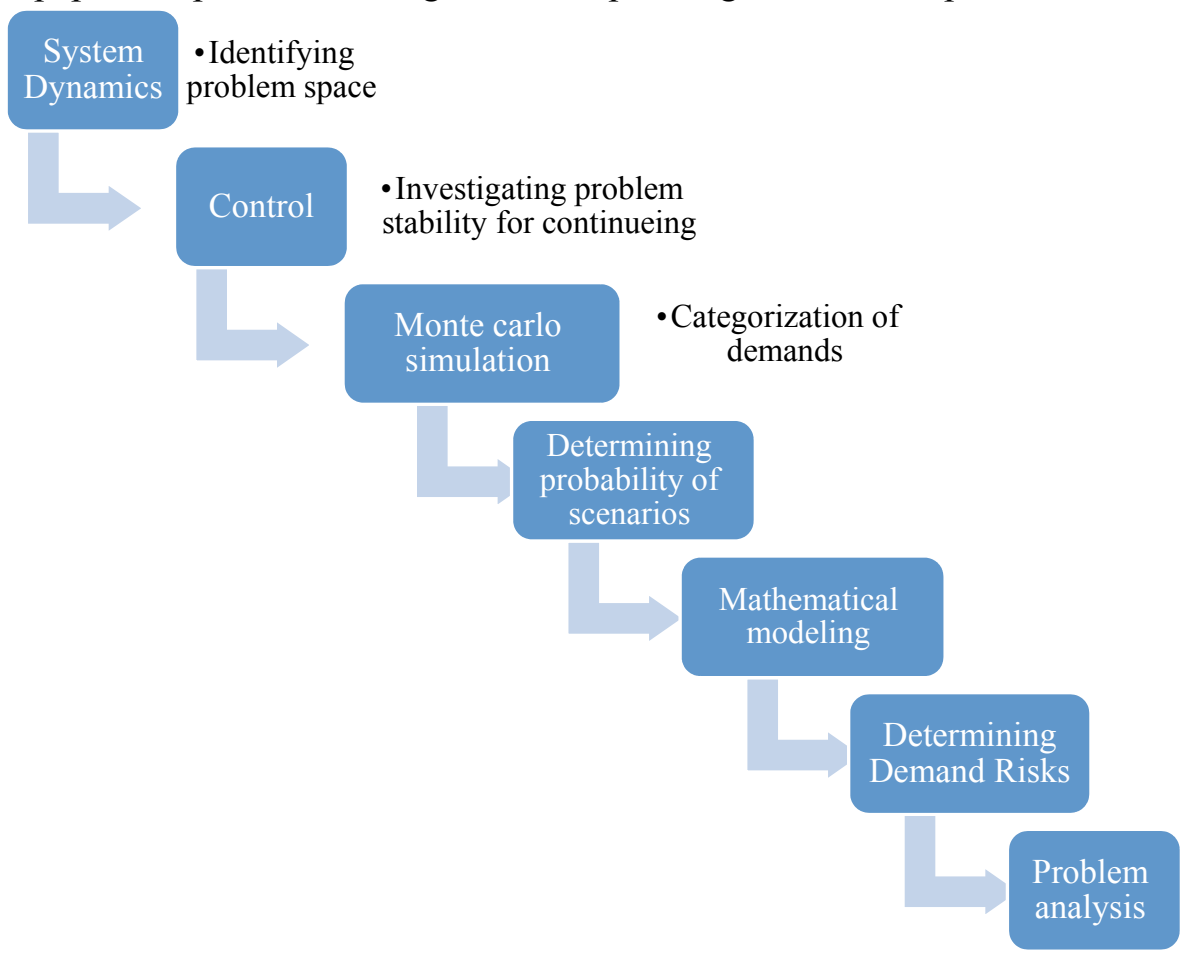

Fig. 1. The proposed solution algorithm 


\subsection{System Dynamics and control}

System dynamics is one of the first responses to the weaknesses of operation research and other techniques of the management science which was presented by Forrester (1961) in MIT University for the first time. To determine the space of the emergency problem which is going to be modeled, causal diagram of system dynamic can be used. With the causal diagram, the flow diagram of emergency problem can be determined by knowing the variables and the level. The diagram in Fig. 2 is the flow diagram of the emergency problem which has been investigated. Structure of a complex one feedback loop system is not simple and in which a state of the system causes the system how to behave. A complex system has many feedback loops. The rates and internal levels of it are non-linear. The complex system is of high order which means that the number of the level variables are high. These systems are usually involved with positive loops which represent the growth processes. Also, it has negative or purposeful loops. As it can be seen in Fig. 2, the problem includes two rates. The input rate which is proportional to the rate of service covering and the cost rate which is proportional to the rate of emergency demand or in other words, the level and number of the patients. This means show the level of unsatisfied demand. The rate of service covering in the real world is rarely equal to the demand and as a result, responsiveness capacity would be a specific percentage of the demand. The responsiveness capacity is also proportional to the intersection of the number of the untreated patients, the minimum capacity of the emergency station and the reception capacity of the hospital. As the constraints generate the feasible area of decision making, the minimum capacity of the hospital and the emergency station is regarded as the basic capacity $\left(c^{\cdot}=\operatorname{Min}(A, B)\right)$.

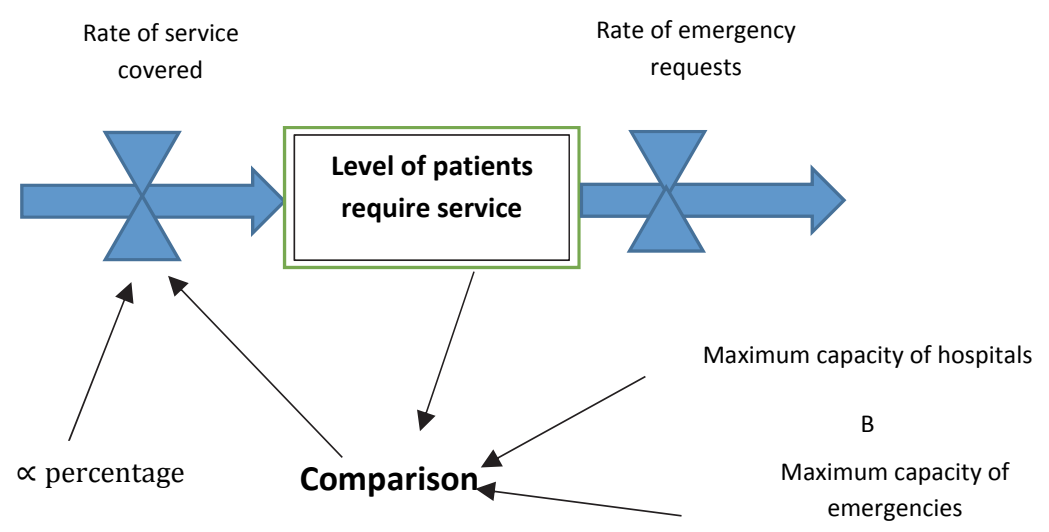

Fig. 2. Proposed flow diagram

In Fig. 3, the block diagram based on the causal diagram is presented. Also, the signal diagram is shown in Fig. 4. These diagrams are adapted from Ogata (2007). Details of applied symbols is presented in Table 1.

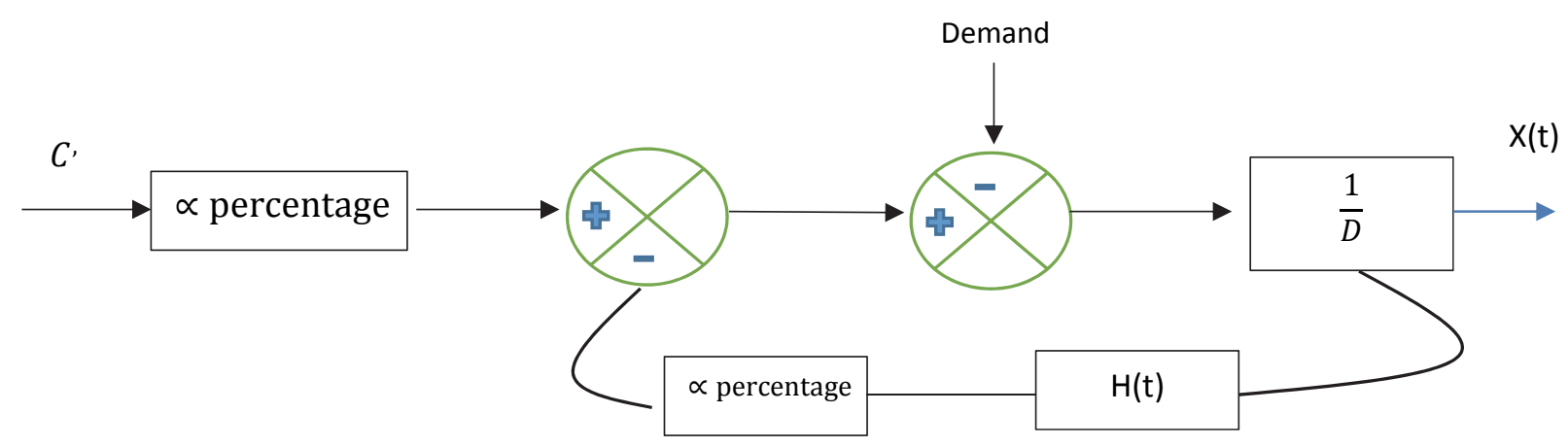

Fig. 3. The block diagram 


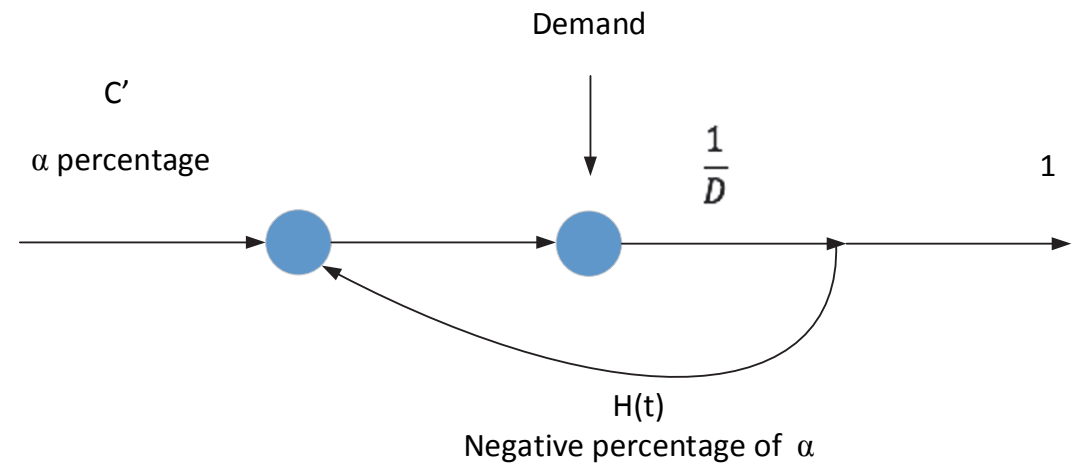

Fig. 4. The signal diagram

Table 1

Details of proposed diagrams

\begin{tabular}{llll}
\hline Symbol & & Mathematical Base & Description \\
\hline & $c$ & $\operatorname{Min}(A, B)$ & Minimum capacity of hospital \\
Demand & & & Demand of injured people \\
$\mathrm{X}(\mathrm{t})$ & & & System feedback in $\mathrm{t}$ \\
& $\propto$ & Coefficient as a percentage \\
$\mathrm{H}(\mathrm{t})$ & & & System feedback in $\mathrm{t}$ \\
& $\frac{1}{D}$ & Integral based on $\mathrm{d} t$ & Update uncovered demands \\
\hline
\end{tabular}

In order to make a decision in such problems and to determine and improve the problem space, it is vital to ensure that the decision space is stable. Because, making decisions in an unknown and highly variable circumstance do not lead to appropriate results. Thus, decision space must be stable in order to consider uncertainty in the problem and make the problem closer to the real world. The control step is presented according to Ogata (2007) and it can be proved that problem is controllable. To investigate the stability of a system, transformation function of the system must be determined. Then, according to the flow equations, blocked and signal equations (which were represented in previous section) the Mason interest can be calculated (see Appendix 1)

\subsection{Simulation}

In this paper, demand is defined by intensity and value. In this way, demand is introduced by six scenarios based on its probability. The considered probability value and the demand intensity are presented in Table 2 .

\section{Table 2}

The probability value and the demand intensity

\begin{tabular}{lrrrrrrr}
\hline Probability & 0.05 & 0.15 & 0.3 & 0.25 & 0.15 & 0.1 & $\sum p_{\mathrm{i}}=1$ \\
\hline $\begin{array}{l}\text { Demand } \\
\text { intensity }\end{array}$ & 5 & 4 & 3 & 2 & 1 & 0 & \\
\hline
\end{tabular}

Using Monte Carlo simulation, the cumulative diagram of Table 1 can be presented. Also, using random numbers corresponding to the scenarios, uncertainty condition is transformed to deterministic one. The results are represented in Table 3:

\section{Table 3}

The certain intensity of demand

\begin{tabular}{lrrrrrr}
\hline Random generated number & 0.23 & 0.58 & 0.57 & 0.78 & 0.26 \\
\hline Intensity of certain demand & 2 & 3 & 3 & 3 & 1 \\
\hline
\end{tabular}


In the Table 4, demand is represented with the probability of the event.

Table 4

The probability and demand values

\begin{tabular}{rllllllll}
\hline Probability & 0.1 & 0.15 & 0.25 & 0.2 & 0.1 & 0.1 & 0.05 & 0.05 \\
\hline Demand & 0.4 & 35 & 30 & 25 & 20 & 0.15 & 10 & 5 \\
\hline
\end{tabular}

Also, by using the Monte Carlo simulation, final results are given in Table 5.

\section{Table 5}

Confirmed values

\begin{tabular}{lll|lll|lll|lll|l}
\hline Random number & 0.21 & 0.4 & 0.15 & 0.74 & 0.1 & 0.6 & 0.69 & 0.4 & 0.28 & 0.74 & 0.75 & 0.85 \\
\hline Intensity & 2 & & 3 & & & 3 & & & 3 & & & 1 \\
Demand & \multicolumn{2}{c}{65} & & 75 & & & 70 & & & 50 & & \\
\hline
\end{tabular}

By doing so, demands are transformed into a deterministic value.

For instance, consider a city which requires relief logistics. A city can be divided into three sections and demand of each section needs to be determined through the above-mentioned approach. While the demand of a section is related to other sections, it can be a coefficient of the specified section with respect to the relation type. For example, if the eastern area of the city is assumed as a host of employee's traffic in the morning, the other areas of this city will be the host at the end of the work shift. Thus, the incident is higher in the morning for the eastern area and it is higher in other areas at the end of the work shifts.

\subsection{Determining the probability of the scenario occurrence}

Scenarios have been extracted from the model below based on two criteria due to the utilities (Wang et al., 2016). A small example has been considered and analyzed by the proposed model of Wang et al. (see Appendix 2). The results for solving the model are shown in Table 6.

\section{Table 6}

The obtained values from the model

\begin{tabular}{ccc}
\hline Values obtained from the model & p \\
\hline 0.6 & 1 & 2 \\
0.4 & 3 & 4 \\
0.4 & 5 & \\
\hline
\end{tabular}

\subsection{Determining Demand Risks}

Data covering analysis appeared at the end of the 70s. Data covering analysis is used as a method to evaluate the performance of decision-making units (DMUs). The data covering analysis is based on linear programming which provides the efficiency of each part without having access to mental and economic values such as weight and the cost (Mavrotas \& Trifillis, 2006). The following model has been used as the basic model to evaluate the efficiency in this paper.

\section{Slacks- Based Measure (SBM) Model}

Data envelopment method was originally developed by Charnes et al. (1987). CCR models, a special form of DEA, under weak efficiency assess the radial (relative) efficiency $\theta^{*}$ and do not consider lack of outputs or excess inputs. This is a shortcoming because $\theta^{*}$ does not consider non-zero auxiliary 
variables. Although the cumulative model is directly dealt with excess inputs and outputs and distinguishes the efficient and non-efficient DMUs, like $\theta^{*}$ in CCR model, it does not measure the depth of non-efficiency. In order to overcome this deficiency, an efficiency measurement method based on auxiliary variables is used as a scalar with a value no less than $\theta^{*}$, that is called Slacks- Based Measure (SBM).

(1) This value is stable to the measuring unit of input and output factors (independent of unit).

(2) This value is uniformly reducing according to the input and output auxiliary variables (uniform). The following fraction can be used to estimate the efficiency of $\left(\mathrm{X}_{\mathrm{O}}, \mathrm{Y}_{\mathrm{O}}\right) \mathrm{DMU}$ :

$$
(S B M) \rho=\frac{1-\frac{1}{m} \sum_{i=1}^{m} s_{i}^{-} / x_{i o}}{1-\frac{1}{s} \sum_{r=1}^{s} s_{r}^{+} / y_{r o}}
$$

s.t.

$$
\begin{aligned}
& \mathrm{x}_{\mathrm{o}}=\mathrm{x} \lambda+\mathrm{S}^{-} \\
& \mathrm{y}_{\mathrm{o}}=\mathrm{Y} \lambda-\mathrm{S}^{+} \\
& \lambda \geq 0, S^{-} \geq 0, S^{+} \geq 0
\end{aligned}
$$

where, $\lambda, \mathrm{S}$ - and $\mathrm{S}+$ are the variables and $X \geq 0$. If $\mathrm{x}_{\mathrm{io}}$ equal to zero, then we will eliminate $\mathrm{S}_{\mathrm{i}}^{-} / \mathrm{x}_{\mathrm{io}}$

from the function. If $y_{i o} \leq 0$, then we will change it with a smaller positive number so that $\mathrm{S}_{\mathrm{r}}^{+} / \mathrm{y}_{\text {ro }}$ will be consider as a penalty value (Cooper et al., 2004).

The application of data envelopment analysis model in this paper is as follows: the emergency demands can be studied in terms of risks. That is, the demand has taken place at what probability that has caused danger and increased the injuries. In such cases, facilities and forces are required to be responsive so that detection and the pace of performance will increase. Therefore, the four criteria including the probability of risk growth, increase of injuries, respondents' equipment in terms of facilities and the required forces to be responsive in the form of humans can be studied for each demand. From the DEA point of view, the first two criteria are inputs and the next two criteria are outputs for risk assessment of each demand. In order to show the increase in demands, the efficiency obtained from each demand risk should be added to 1 number, so that the increase in responsiveness for controlling the risk can be shown in demands. On the other hand, some of these demands are discussable as control tools over inputs and some other on outputs. Therefore, the efficiency of some demands can be obtained from input-centered models and some others from output-centered models. Some of the demands are dependent on demands that are not discussable in the problem or factors that are not directly related to the event in kind and entity. Hence, they affect the uncovered demand. That is why they should be considered in determining the efficiency so that demand risks can be analyzed more realistically.

\subsection{Presenting the model}

In current research, integration of planning of relief supply chain which includes the location of the stations, assigning ambulances to them and also ambulances routing to relieve the patients in a standard time and with the minimum cost is considered. To model the problem, consider a city that needs to be relieved when a disaster occurs. Problem can be shown as a graph network therefore, some nodes of the graph (in the considered city) are candidate locations for building and establishing the stations for the ambulances and also, stations can be established with various costs in these nodes. These centers are depot of the ambulances where they begin their tour from there to go to the required nodes and then, to the hospitals. So, different paths can be chosen. The optimal routes of ambulances is in line with optimal objective value. Traffic load of these paths depends on the time and because of this, a single 
approach cannot be chosen for all times. As a consequence, different scenarios should be simulated. This traffic load has a direct impact on the traveling time. It is possible that a long time would be necessary for a short path and vice versa. Therefore, it is more appropriate to consider time instead of the path between two points. Finally, it should be decided about the selection of destination hospitals. The possibility of transferring the ambulances in order to have a better use of available resources is also considered. On the other hand, due to the uncertainty of demand of ambulances in the stations, demands are considered based on different scenarios.

The proposed model has multiple steps that in one step the assignment is based on the scenarios for satisfying the demand of emergency stations. In next step, the final assignment for establishing the ambulances in the stations based on the assigned scenario is conducted and then based on the final assignment, the locations of the needed emergencies are established. Finally, after locating the emergencies centers, the routes are constructed proportional to them. Therefore, the proposed model is a multi-step one. In next section, the model is presented.

A schematic example has been depicted in Fig. 5. Six applicants, one hospital and two potential emergency stations are considered in one section city with. The constructed routes are shown using arrows toward demand nodes. Only one of two potential emergency stations is established with respect to demands value.

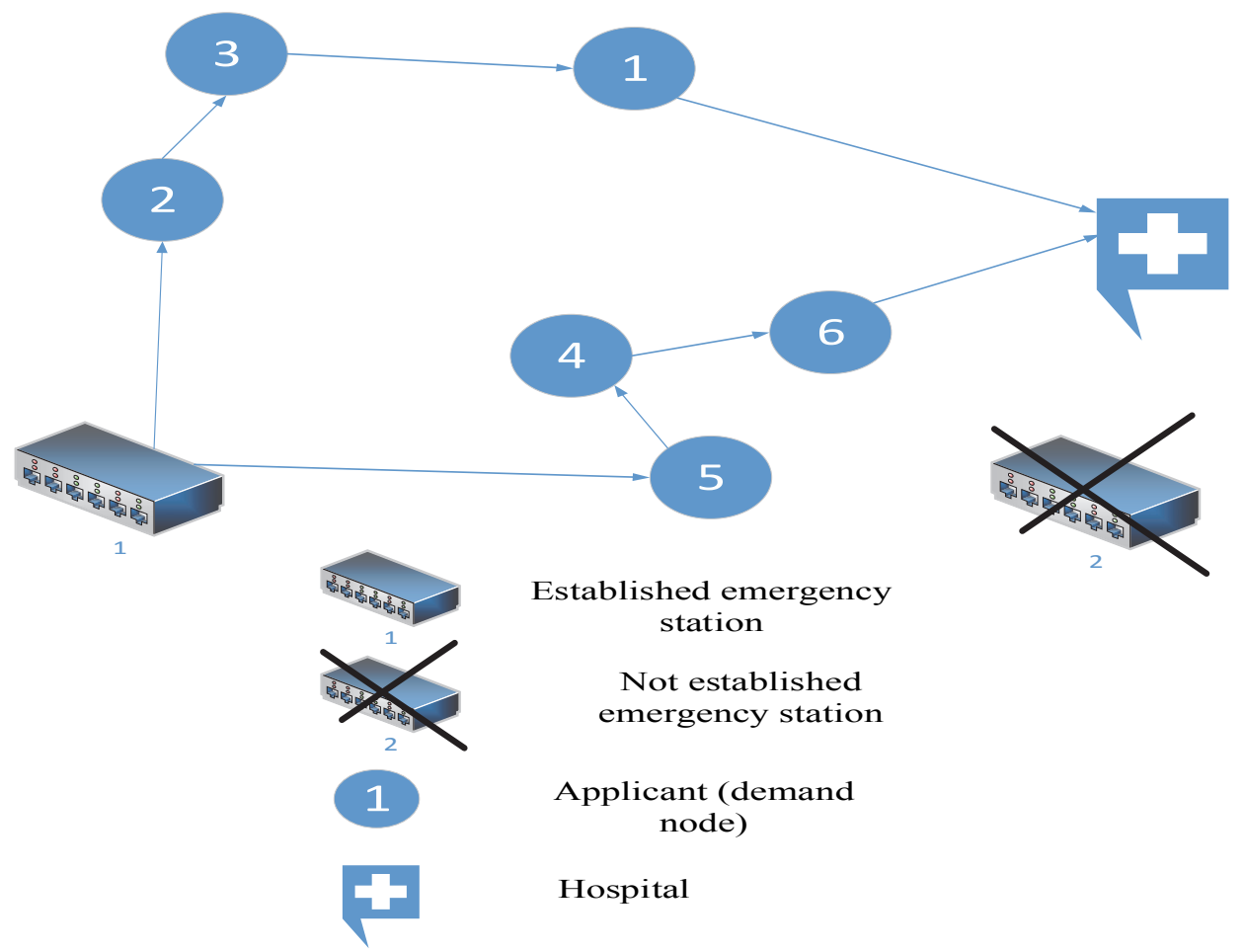

Fig. 5. The schematic example

After simulating the demand and determining the risk according to SBM, the proposed models can be presented.

\section{Indices}

$i$ : applicants

$j$ : emergency stations

$k$ : hospitals

$S$ : scenarios 


\section{Parameters}

$d_{i s}$ : The quantity of demand $\mathrm{i}$ in scenario $\mathrm{S}$

$\mathrm{T}$ : The standard time

$\alpha$ : Minimum cover percentage

$\mathrm{t}_{\mathrm{ijk}}$ : The traversing time from emergency station $\mathrm{j}$ to the demand $\mathrm{i}$ and from then, to the hospital $\mathrm{k}$ M: A large number

$\mathrm{f}_{\mathrm{j}}$ : Establishing cost for emergency location $\mathrm{j}$

$\mathrm{g}_{\mathrm{j}}$ : Cost of assigning the ambulance to the emergency station $\mathrm{j}$

$\mathrm{C}_{\mathrm{s}}$ : Cost of required ambulance for demand $\mathrm{i}$ from the station $\mathrm{j}$ under the scenario $\mathrm{S}$

A: Cost of establishing a path from $\mathrm{i}$ to $\mathrm{j}$ up to $\mathrm{k}$

$\mathrm{B}$ : Cost of establishing a special path from $\mathrm{i}$ to $\mathrm{j}$ and to $\mathrm{k}$

Bb: budget

$b_{S}$ : Performance coefficient for assignment in each scenario

$\omega$ : Coefficient of feasible space

$\lambda$ : Coefficient of scenario effects

M: A large number

dd: The difference between fuzzy central number with the lower bound

$\theta$ : Confidence percentage

$\overline{d_{l s}}$ : The average of demands

$\tilde{C}$ : The cost of fuzzy assignment

$\tau_{s}:$ Entrance rate of each demand in each scenario

$\vartheta_{s}$ : The mean time of servicing in each scenario

$\pi_{i s}$ : The efficiency value obtained from data enveloping analysis model for demand i under scenario $\mathrm{s}$

\section{Decision making variables}

$X j: 1$, if location $\mathrm{j}$ is established as the emergency station, otherwise 0 .

$y_{i j s}$ : The number of needed ambulance for demand $\mathrm{i}$ in station $\mathrm{j}$ under scenario $\mathrm{S}$

$Z_{j}$ : The number of assigned ambulances to the emergency station $\mathrm{j}$

$x_{i j k}: 1$, if the ambulance goes from emergency $\mathrm{j}$ to the demand $\mathrm{i}$ and then to the hospital $\mathrm{k}$, otherwise 0 .

$x_{i j k}^{\prime}: 1$, if the ambulance goes from emergency $\mathrm{j}$ to the demand $\mathrm{i}$ and then to the hospital $\mathrm{k}$ through a special path, otherwise 0 .

$V_{i j s}, V$ : the transformer variable

$Q u_{i s}:$ the number of ambulances giving services to each demand in each scenario

\subsection{Robust model}

For retrofitting the model in order to do the final assignment of the ambulance according to the number of required ambulances for each demand point, the deviation from the feasible space is considered. On the other hand, the impact of other scenarios on each scenario is considered as a retrofitting factor for the model and in the objective function. This means in each scenario the number of the needed ambulance in each demand point causes the differences with the other scenarios.

$$
\begin{array}{r}
\operatorname{Min} \sum_{j}\left(f_{j}, x_{j}+g_{j}, z_{j}\right)+\sum_{i} \sum_{j} \sum_{k}\left(A x_{i j k}+B x_{i j k}^{\prime}\right)+\sum_{s} \sum_{i} \sum_{j} p_{s} y_{i j s} \\
+\lambda \sum_{s} p_{s}\left(\sum_{i} \sum_{j} y_{i j s}-\sum_{s} p_{s} \sum_{i} \sum_{j} y_{i j s}+2 \theta_{s}\right)+w \sum_{s} p_{s}\left(\delta_{s}+2 \Delta_{s}\right)
\end{array}
$$




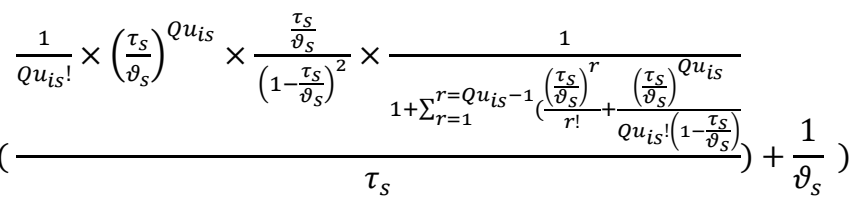

$$
\begin{aligned}
& -M x j+z j \leq 0 \quad \forall_{j} \\
& \sum_{i} y_{i j s}-b_{s} z_{j}+\delta_{s}=0 \quad \forall_{j, s} \\
& \alpha \sum_{i} \sum_{s} d_{i s}-\sum_{i} \sum_{j} \sum_{s} y_{i j s} \leq 0 \\
& \sum_{j} y_{i j s}-d_{i s} \leq 0 \quad \forall_{i, s} \\
& t_{i j k} x_{i j k}-M x_{i j k}^{\prime}-T \leq 0 \quad \forall_{i, j, k} \\
& x_{j}-\sum_{i} \sum_{k} x_{i j k} \leq 0 \quad \forall_{j} \\
& y_{i j s}-\sum_{k} x_{i j k} \leq 0 \quad \forall_{i, j, s} \\
& -\theta_{s}-\sum_{i} \sum_{j} y_{i j s}+\sum_{s} p_{s} \sum_{i} \sum_{j} y_{i j s} \leq 0 \quad \forall_{s} \\
& -\Delta_{s}-\delta_{s} \leq 0 \quad \forall_{s} \\
& \sum_{i} \sum_{k} x_{i j k} \leq M z_{j} \quad \forall_{j} \\
& \sum_{j} y_{i j s} \leq Q u_{i s} \quad \forall_{i}, \forall_{s} \\
& y_{i j s} \geq 0, Z_{j} \geq 0, Q u_{i s} \geq 0 \text { integer }, \forall_{i, j, s} ; \quad x_{i j k}, X_{j}, x_{i j k}^{\prime} \in\{0,1\} \quad \forall_{j} ; \Delta_{s}, \theta_{s} \geq 0 \quad \forall_{s}
\end{aligned}
$$

Objective function (1) includes five terms. The first term minimizes the cost of establishing the emergency location and the cost of final assignment of ambulance to the emergency station. The second term tries to minimize the cost of establishing the path and the special path. The third term aims to minimize the number of needed ambulances in each scenario. The fourth term represents the impact of other scenarios on the other ones. The fifth term investigates the feasibility of the problem. The last term tries to minimize the length of the generated queue. This queue contains time rate between two arrivals, service time rate, and $m$ service providers. In simple terms queue of type $\mathrm{M} / \mathrm{M} / \mathrm{m}$ at steady state is assumed. Minimizing queue length leads to faster response in relief logistics, and this is the reason why we consider it. The amount of allocated resources of the mean was calculated from Poisson distribution which is estimation by confidence interval.

When the assignment of ambulances to the stations is done, a location must be established in that place as a station (Eq. (2)). The final assignment of a location is directly linked with the number of ambulances that is demanded in each scenario (Eq. (3)). The total number of ambulances that are required in each scenario should be at least $\alpha$ percent of the total demand (Eq. (4)). The number of required ambulances for each demand in each scenario would be less than the demand (Eq. (5)). The total time that a path is formed must be less than the standard time $T$. Otherwise, the path will transform to a special path (Eq. (6)). In simple words, when the total time for the process of going from the emergency station up to carrying injured people to the hospital is more than the standard time (which is calculated by the experts based on the probability of injured survival) then, a special path of 
emergency is provided which reduces the time in terms of management and physical structure. In management terms, it is based on the traffic control of the region and in structural terms, it can help be by establishing an emergency path. The path is constructed when the emergency station is established (Eq. (7)). The path is constructed according to the number of assigned ambulances (Eq. (8)). Eq. (9) and Eq. (11) are the limitations of robust linearization. Eq. (11) guarantees the formation of a path of ambulance station. Eq. (12) determines the number of servicing ambulances in each scenario. Type of variables is specified in Eq. (14).

\subsection{Mathematical Model for allocation of uncertain servicing resources}

In this section, the required amount for each demand point of emergency location is considered stochastically and also, supplier resources are dealing with uncertainty in meeting the requirements. In other words, each demand point tries to maximize the probability of satisfying its own emergency needs due to the fact that the resources which employ are dealt with uncertainty.

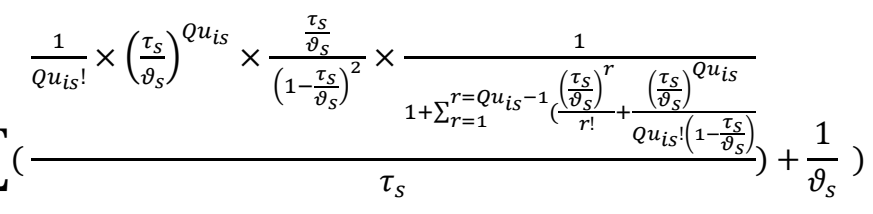

$$
\begin{aligned}
& -M x j+z j \leq 0 \quad \forall_{j} \\
& \sum_{i} y_{i j s}-z_{j} \leq 0 \quad \forall_{j, s} \\
& \alpha \sum_{i} \sum_{s} d_{i s}-\sum_{i} \sum_{j} \sum_{s} y_{i j s} \leq 0 \\
& \sum_{j} y_{i j s}-d_{i s} \leq 0 \quad \forall_{i, s} \\
& t_{i j k} x_{i j k}-M x_{i j k}^{\prime}-T \leq 0 \quad \forall_{i, j, k} \\
& x_{j}-\sum_{i} \sum_{k} x_{i j k} \leq 0 \quad \forall_{j} \\
& y_{i j s}-\sum_{k} x_{i j k} \leq 0 \quad \forall_{i, j, s} \\
& \sum_{j}\left(f_{j} x_{j}+g_{j} z_{j}\right)+\sum_{i} \sum_{j} \sum_{k}\left(A x_{i j k}+B x_{i j k}^{\prime}\right)+\sum_{i} \sum_{j} \sum_{s} y_{i j s} \leq B b \\
& \sum_{j} y_{i j s} \leq \mu \bar{f} \quad \forall_{i, s} \\
& \sum_{i} \sum_{k} x_{i j k} \leq M z_{j} \quad \forall_{j} \\
& \bar{x}-Z_{\frac{\gamma}{2}} \sqrt{\frac{\bar{x}}{n}} \leq \mu \leq \bar{x}+Z_{\frac{\gamma}{2}} \sqrt{\frac{\bar{x}}{n}} \\
& \sum_{j} y_{i j s} \leq Q u_{i s} \quad \forall_{i}, \forall_{s}
\end{aligned}
$$

The objective function aims to increase the allocation probability of ambulance to the locations that satisfy the demand in each scenario (Eq. (14)). Eqs. (15-21), Eq. (24) and Eq. (25) are the same as before. This constraint shows that the financial resources (budget) is always a fixed sum (Eq. (22)). 
Eq. (24) shows that the total required ambulances in each scenario for each demand point follows the average of Poisson's distribution. The impetus behind using this distribution is that the occurrence probability of a number of these event is related to the time duration and also, number of events in separated time intervals are independent to each other. Eq. (25) is the confidence distance for $\mu$ in Poisson's distribution. In this constraint, $\bar{x}$ is the average, $n$ is the sample size and $\bar{X} \pm Z_{\frac{\gamma}{2}} \sqrt{\frac{\bar{x}}{n}}$ is the onesided confidence level with the confidence level of $(1-\gamma) \%$. Type of the variables is specified in constraint (27).

\subsection{Robust model with uncertainty servicing resources and considering the probability of satisfying the fuzzy demand with fuzzy costs}

In this model, sum of the assigned costs to the ambulance of each point is proportional to the location of emergency stations and it is considered as a fuzzy triangular. Therefore, we aim to increase the responsiveness chance for the needs of each demand point. Moreover, the assigned cost to the ambulance in each demand point is proportional to the emergency stations and it is considered as fuzzy triangular number. Hence, the model tries to increase the chance of responsiveness to the needs of each demand point.

$$
\begin{aligned}
& \operatorname{Max} \bar{f}-\sum_{i} \sum_{j} \sum_{s} p_{s} y_{i j s}-\lambda \sum_{s} p_{s}\left(\sum_{i} \sum_{j} y_{i j s}-\sum_{s} p_{s} \sum_{i} \sum_{j} y_{i j s}+2 \theta s\right) \\
& -w \sum_{s} p_{s}\left(\delta^{2}+2 \Delta s\right)-\sum_{s} \sum_{i} \sum_{j} V_{i j s}
\end{aligned}
$$

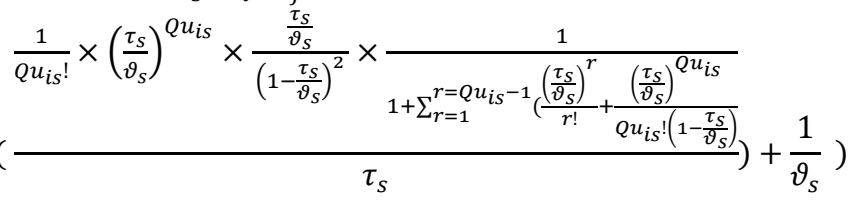

$$
\begin{aligned}
& \text { subject to } \\
& -M x j+z j \leq 0 \quad \forall_{j} \\
& \sum_{i} y_{i j s}-b_{s} z_{j}+\delta_{s}=0 \quad \forall_{j, s} \\
& \alpha \sum_{i} \sum_{s} d_{i s}-\sum_{i} \sum_{j} \sum_{s} y_{i j s} \leq 0 \\
& \sum_{j} y_{i j s}-d_{i s} \leq 0 \quad \forall_{i, s} \\
& \emptyset_{(\theta)}^{-1} y_{i j s} \geq d_{i s}-\overline{d_{l s}} \quad \forall_{i, j, s} \\
& y_{i j s} \leq(1-2 \theta)\left(d_{i s}-d d\right)+2 \theta d_{i s} \quad \forall \forall_{i, j, s} \\
& t_{i j k} x_{i j k}-M x_{i j k}^{\prime}-T \leq 0 \quad \forall_{i, j, k} \\
& x_{j}-\sum_{i} \sum_{k} x_{i j k} \leq 0 \quad \forall_{j} \\
& y_{i j s}-M \sum_{k} x_{i j k} \leq 0 \quad \forall_{i, j, s} \\
& -\theta_{s}-\sum_{i} \sum_{j} y_{i j s}+\sum_{s} p_{s} \sum_{i} \sum_{j} y_{i j s} \leq 0 \quad \forall_{s} \\
& -\Delta_{s}-\delta_{s} \leq 0 \quad \forall_{s} \\
& \sum_{i} \sum_{j} \sum_{s} V_{i j s}=V
\end{aligned}
$$




$$
\begin{aligned}
& \sum_{i} \sum_{j} \sum_{s} E(\tilde{c}) p_{s} y_{i j s} \leq V \\
& \sum_{j}^{j}\left(f_{j} x_{j}+g_{j} z_{j}\right)+\sum_{i} \sum_{j} \sum_{k}\left(A x_{i j k}+B x_{i j k}^{\prime}\right)+\sum_{i} \sum_{j} \sum_{s} y_{i j s} \leq B b \\
& \sum_{j}^{j} y_{i j s} \leq \mu \bar{f} \quad \forall_{i}, \forall_{s} \\
& \sum_{i} \sum_{k} x_{i j k} \leq M z_{j} \quad \forall_{j} \\
& \bar{x}-Z_{\frac{\gamma}{2}} \sqrt{\frac{\bar{x}}{n} \leq \mu \leq \bar{x}+Z_{\frac{\gamma}{2}} \sqrt{\frac{\bar{x}}{n}}} \\
& \sum_{j} y_{i j s} \leq Q u_{i s} \quad \forall_{i}, \forall_{s} \\
& \bar{f} \quad \text { free variable, } \\
& Z_{j} \geq 0, X_{j} \in\{0,1\}, x_{i j k}, x_{i j k}^{\prime} \in\{0,1\}, \Delta_{s}, \theta_{s} \geq 0, V_{i j s}, V \geq 0 \quad Q u_{i s} \geq 0 \quad \text { integer }, \forall_{i, j, s}
\end{aligned}
$$

The objective function has five terms (Eq. (32)). The first term tries to increase the probability of allocation of the ambulance to the locations that satisfy the demand in each scenario. The second term minimizes the number of required ambulances in each scenario. The third and fourth terms are adopted from the main components of robust model. The third term represents the effect of other scenarios on the current scenario. The fourth term investigates the feasibility of the problem. The fifth term focuses on the minimization of the number of ambulances in order to minimize the costs. The number of required ambulances for each demand in each scenario is less than the demand (Eq. (36)). For demand, the chance of being a proportion of the demand point in each scenario from each location is based on the distribution probability. Here, the distribution for the probability coefficients of the variable is based on the normal distribution with the average of zero and variance of one (Eq. (37)). For demand, the chance of being a proportion of the demand point in each scenario from each location is based on the fuzzy demand. For this constraint, it is assumed that the maximum confidence is 50 percent (Eq. (38)). To solve the problem, the minimum of the Eq. (37) and Eq. (38) are considered. The solution in both of the problems is feasible but the better problem can be selected according to it conditions.

\section{Solution method}

In this section, a heuristic and a metaheuristic algorithm (namely Simulated Annealing (SA)) are developed to solve the proposed model. The heuristic is applied to generate initial solutions and the SA is applied to improve these solutions.

\subsection{Solution Representation}

To show the binary variables, we used a single-row matrix that is related to emergency station locations. Its dimension is $1 \times|I|$ in which columns are potential locations for establishing the emergency station. This matrix's elements are zero and one in a way that zero and one correspond to establishment and non-establishment of the emergency station.

$$
X_{j}=\left[\begin{array}{llll}
X_{1} & X_{2} & \ldots & X_{j}
\end{array}\right]
$$

For example, the solution representation for this matric can be written as Fig. 6 if four candidate locations exist for establishing the transfer points.

\begin{tabular}{|l|l|l|l|}
\hline \multicolumn{4}{|c|}{ Emergency station } \\
\hline 0 & 0 & 0 & 1 \\
\hline
\end{tabular}

Fig. 6. Solution representation for initial solution 
After that, a constructive heuristic algorithm is used to generate initial servicing routes for ambulances. The steps of algorithm are as follows:

1- Assign ambulances to the established station. Assign a demand point to the ambulance randomly.

2- Select next demand point with respect to the related constraints such as considering standard time to service. In addition, we should select that demand point which has the least cost of establishing a path and least traversing time. If we have multiple points satisfy these constraints, select one of these randomly and service it.

3- If standard time limitation has been violated, send another ambulance. Otherwise, service other demand points.

4- If all demand points are being serviced, go to 5. Otherwise go to 2.

5- Stop the algorithm. Report the solution.

The solution representation for the constructed routes of ambulances in a station is presented in Fig. 7. We have some ambulances assigned to the established stations. Each row represents constructed tour of the ambulances. If we have no demand point to service, zero is put in the matrix.

\begin{tabular}{|c|c|c|c|c|c|c|c|c|}
\hline \#Ambulance in established station & \multicolumn{9}{|c|}{ Demand point } & hospital \\
\hline 1 & 2 & 5 & 6 & 4 & 0 & 0 & 0 & 1 \\
\hline 2 & 1 & 7 & 3 & 0 & 0 & 0 & 0 & 1 \\
\hline
\end{tabular}

Fig. 7. Solution representation for initial solution

\subsection{Simulated Annealing}

Simulated annealing is a technique which have been applied to problems that are both difficult and important. The simulated annealing begins its search from a random initial solution. The iteration loop that characterizes the main procedure randomly generates in each iteration only one neighbor $s$ ' of the current solution $s$. The variation $\Delta$ for the value of the objective function $(x)$ is tested for each neighbor generation (Hwang, 1998). To test this variation, $\Delta=\left(s^{\prime}\right)-f(s)$ is obtained. If the value of $\Delta$ is less than zero, then the new solution $s$ ' will be automatically accepted to replace $s$. Otherwise, accepting the new solution $s$ ' will depend on the probability established by the Metropolis criteria, which is given by $(-\Delta / T)$, where $T$ is a temperature parameter, a key variable for the method. Therefore we have:

$$
P(\text { Acceptance })=e^{-\frac{\Delta f}{T}}
$$

Fig. 8 shows the simulated annealing pseudocode.

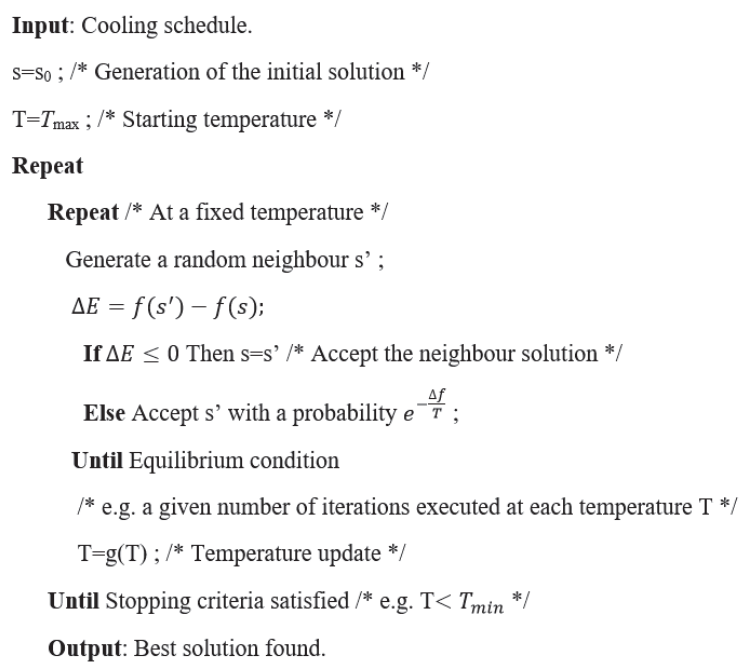

Fig. 8. Simulated annealing pseudo code 
Local searches used in this algorithm are as below:

1. Change the established station to non-established station and choose another one to be established.

In constructed routes by ambulances:

2. Reverse the sequence of the served demand point in each solution.

3. Select a part of a solution is selected randomly and change its sequence.

It is noticeable that feasibility of the new generation of solutions is checked after applying these local searches.

\subsection{Algorithm Stop Criterion}

It has considered a maximum number of iterations for stopping the algorithm. In addition, another stopping criterion is considered as the maximum number of iterations with no improvement for the increasing the efficiency and decreasing waste time. Finally, report the solution with best objective value.

\section{Results analysis}

In first step, we generate two instances in different sizes to evaluate the performance of the proposed algorithm. Then these two instance are solved both by the proposed algorithm and by GAMS software. Instances information are shown in Table 8. It is worth to say the model were analyzed through the GAMS and Baron Solver software in 1000 seconds. The obtained result is presented in Table 7.

\section{Table 7}

The Obtained Results from solving instance problems

\begin{tabular}{llllll}
\hline Instances & GAMS Objective & SA Objective & GAP (\%) & CPU (seconds) & SA Run time (seconds) \\
\hline Small-Sized & -1655.126 & -1673 & 1.07991778 & 659.15 & 13.26 \\
Large-Sized & -5734.900 & -5390 & 0 & 1000 & 36.19 \\
\hline
\end{tabular}

\section{Table 8}

\section{Data for sample problem}

\begin{tabular}{lll}
\hline Parameters & Small-sized instance problem & large-sized instance problem \\
\hline Number of applicants & 10 & 25 \\
Number of potential emergency stations & 4 & 6 \\
Number of hospital & 3 & 5 \\
Number of scenario & 3 & 6 \\
$\mathrm{~T}$ & 100 & 200 \\
$\omega$ & 0.7 & 0.7 \\
$\lambda$ & 0.3 & 0.3 \\
$\emptyset_{(\theta)}^{-1}$ & 1.64 & 1.64 \\
$\mathrm{~d}_{1 \mathrm{~s}}$ & 90 & 90 \\
$\theta$ & 0.2 & 0.2 \\
$\tau_{s}$ & $(20,14.12,8,10)$ & $(20,14.12,8,10)$ \\
$\vartheta_{S}$ & $(22,18,14,10,6)$ & $(22,18,14,10,6)$ \\
$\bar{x}$ & 600 & 600 \\
$\mathrm{n}$ & 100 & 100 \\
$\mathrm{dd}$ & 10 & 10 \\
\hline
\end{tabular}

As it can be seen in Table 7, SA has shown appropriate performance in comparison with GAMS software. It has so much better results in large-sized instance. In the following, we apply a sensitivity analysis on small-sized instance to investigate the effect of uncertainties. Due to the fact that hessian matrix is the limitations of the feasible area and the positive semi-definite objective function and also the problem is linear therefore, the feasible area of the problem and the objective function are convex. 
Hence, in this convex planning type, the problem involves the optimum point which is attained by solving the problem. In other words, the solution is convergent to the optimum point. After demand simulation and its risk determination through SBM, the problem can be analyzed. In fact, the model was solved and analyzed by considering four candidate location for the emergency stations to satisfy three demand points and to transfer the injured to three hospitals. The problem is assignment scenario based on fuzzy demand. Definitions of sets and parameters for the sample problem shown in Table 8 in details. Also, the parameters are generated randomly.

Problem 1 with the limitation of assignment scenario is based on the probability satisfaction of the demands and problem 2 is an assignment scenario based on the fuzzy demand. Parameters of $\omega, \lambda, \emptyset_{(\theta)}^{-1}, \overline{d_{1 s}}$ are changed in the interval of 20 percent decrease and 20 percent increase in order to investigate the sensitivity of the problem to them. Therefore, in Table 9 and figures below, their effect on the objective function and main variables of the problem is investigated. The numerical results are shown in Table 9.

Table 9

Investigating the changes of parameters

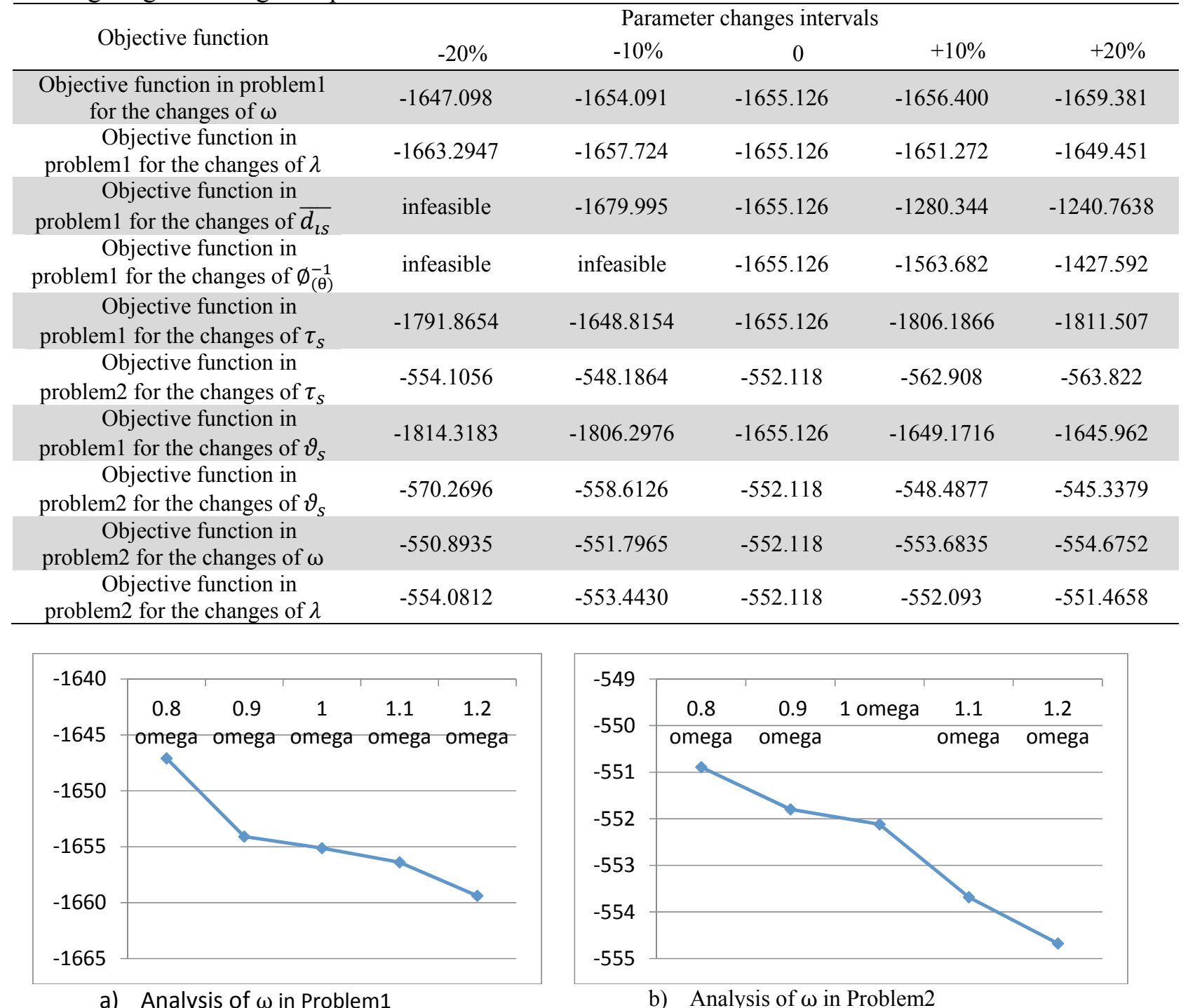




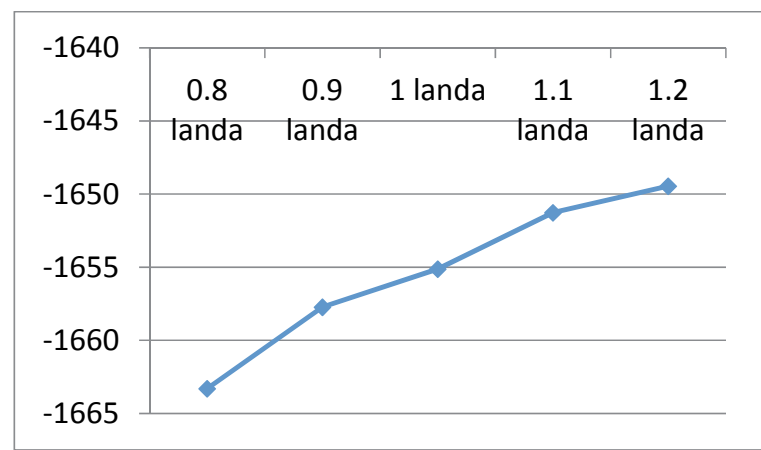

c) Analysis of $\emptyset_{(\theta)}^{-1}$ in Problem1

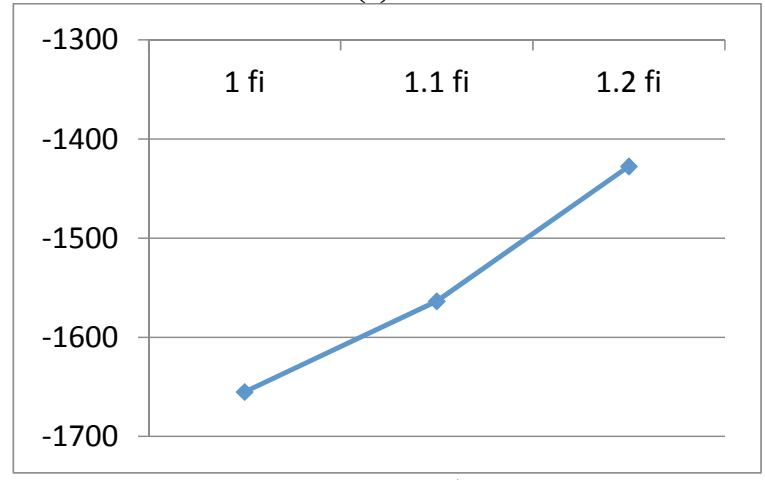

e) Analysis of $\emptyset_{(\theta)}^{-1}$ in Problem2

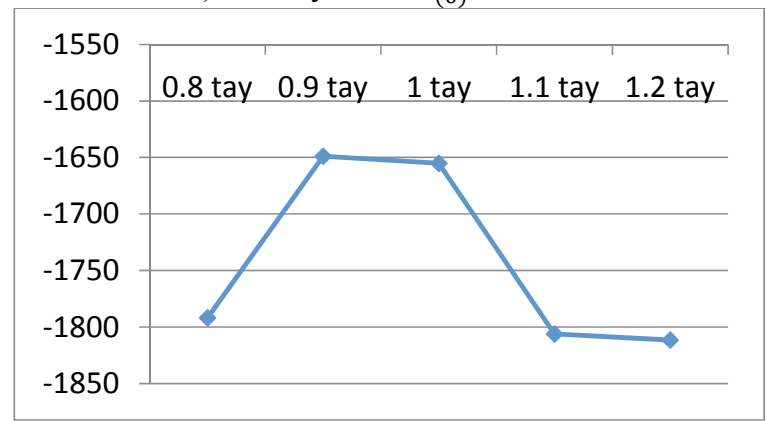

g) Analysis of $\tau_{s}$ in Problem1

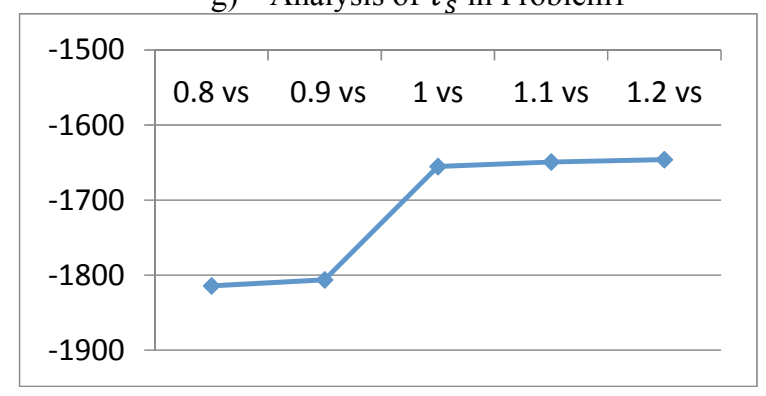

i) Analysis of $\vartheta_{s}$ in Problem1

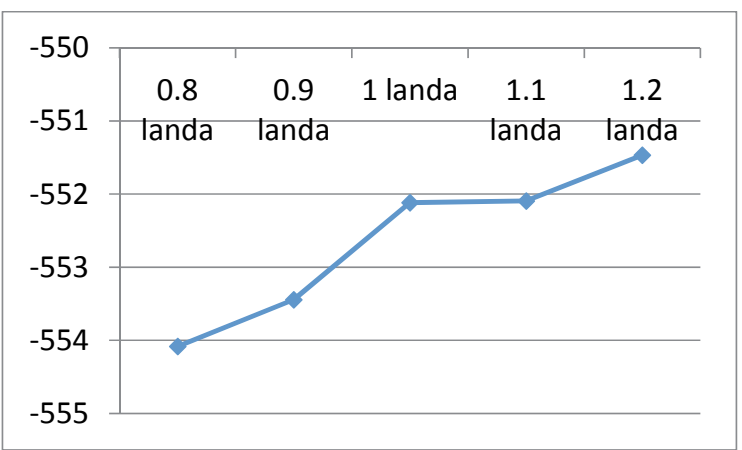

d) Analysis of $\lambda$ in Problem1

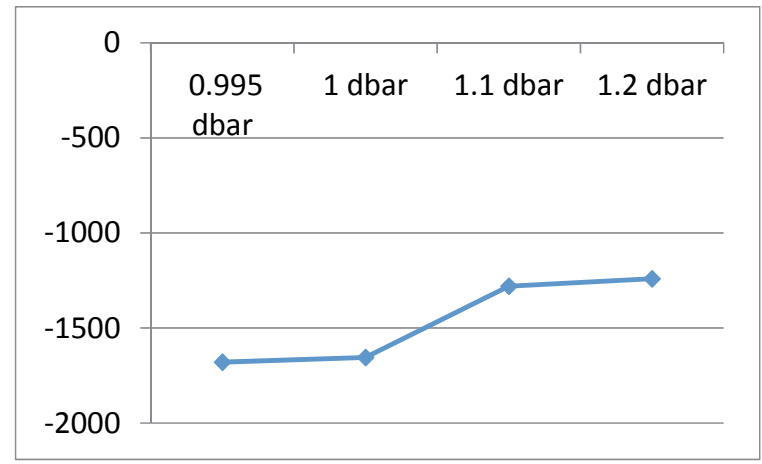

f) Analysis of $\overline{d_{l s}}$ in Problem2

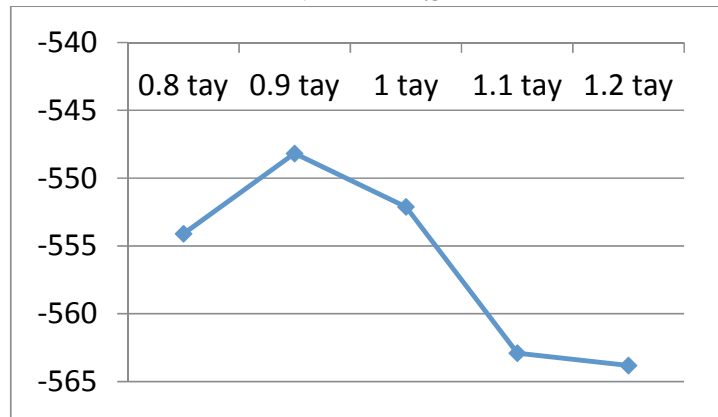

h) Analysis of $\tau_{s}$ in Problem1

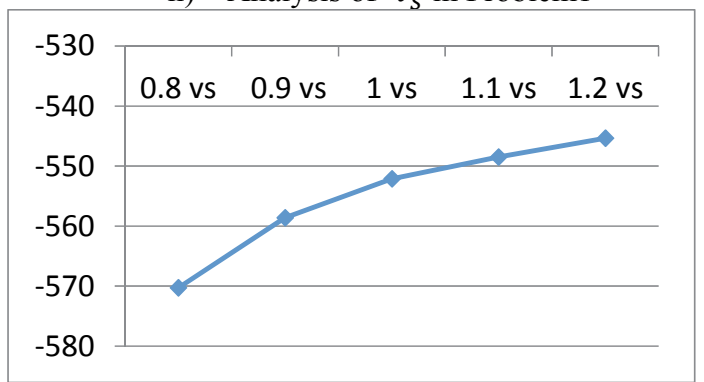

j) Analysis of $\vartheta_{s}$ in Problem1

Fig. 9. Investigating the object function of the problem1 against the changes of the feasible space

As it is represented in Fig. 6, by increasing and decreasing the parameters, objective in problems 1 and 2 represent different manners. For example, by increasing $\omega$, total profit decreases in both problems. It can be mentioned, by inceasing the assignment of ambulance to the locations, the feasible area changes more which leads to the increase of costs (Fig. $9(a, b)$ ). And for the last part of Fig. 9 (Fig. 9 $(i, j)$ ), by increasing $\vartheta_{s}$, the total profit increases in both problems and behaves like $\sqrt{x}$ function in problem 2. By the increase in servicing rate of the ambulances, fewer ambulances will be allocated to 
each station and the costs will decrease. This decrease continues until it reaches the maximum efficiency of each ambulance.

Considering the obtained results and total cost that needs to be minimized so that demands can be met better, when the servicing resources in the first problem that probably meet demands, incur more costs, by the increase in scenario controlling, in general the costs decrease and the model becomes more stable. On the other hand, by the increase in the rate of ambulance servicing, the efficiency of the ambulances increase and this fact leads to the reduction in the final costs. By the increase in the rate of demands, besides the increase in ambulance efficiency, more ambulances should be allocated to each station which will result in an increase in the costs. Hence, the analyzed Fig. are sometimes in fixed slopes, ascending and descending slopes at other times. Generally, if we are seeking to make quick decisions with desirable and more results, we can analyze the aforementioned Figure in line with the objectives and with higher slopes because sometimes the decisions lead to few or negligible outcomes in line with the objectives for the financial and intellectual capitals. Therefore, these losses can be prevented considering the above analyses. Decision makings with quick and desirable outcomes require facilities that sometimes are not available due to environmental restrictions. As a result, decisions with fewer outcomes can be selected using the above analyses so as to gradually reach the desirable point.

\section{Conclusion}

In this paper, the critical problem and relief logistics are investigated through seven steps. In the first step, with using system dynamics, the relief problem space is specified. In the second step, the causal space from control viewpoint is investigated. In the third step, by using simulation, demand the ambulances is attained. In the fourth step, the probability of the occurrence of scenario events with regards to the game theory and according to the traffic and population density is calculated. In the fifth step, asking the experts' opinions, the candidate emergency location have been evaluated and according to their opinions, the models proposed to assess the ability of satisfying the expectations of the decision maker and also, make justifications that decision maker can understand with what amount of deviations he can reach his opinion in the feasible space when there is no response to his expectations. In the sixth step, the integrated multi step modeling of location the emergency stations, allocation and routing of the ambulance according to fuzzy and probability satisfaction of demand was studied, a Simulated annealing algorithm is developed to solve the proposed model. Other innovation of this model include shortening the queue length of emergency applicants which will increase their survival and the higher the rate of servicing is, the better the ambulance efficiency will be. Also, considering uncertain resources for supplying the demand and fuzzy assignment costs with using the robust programming were investigated by SA and GAMS software. Results show that our proposed SA has appropriate performance, especially in large-sized problem. In the seventh step, the problem was analyzed in terms of the obtained results. According to the obtained results, the higher the probability of meeting demands is, the higher costs will be incurred by the objective function. Hence, more demands will be met and if the model is assumed to be met by fuzzy demands, the costs will decrease due to the reduction in responding. In general, the presented algorithm can make the best decisions proportionate to the available conditions apart from recognizing the problem step by step. For future researches some suggestions are recommended as follows:

- Locating emergency centers in moving manner in various periods

- Classifying ambulances according to their services

- Considering ambulances with supportive services for the problem. By sensitivity analysis, the number of supportive ambulances in each emergency location with regards to the required demand is determined to increase the confidence coefficient.

- Specifying distribution of usage of ambulances, so that emergency locations can lend their idle ambulances to the locations with higher demand. 
- Considering ambulance and path maintenance, so that the accessibility to these resources is determined.

- For more justice, an objective function can be defined to decrease the unsatisfied demand.

\section{Appendix 1. Control}

According to Ogata's book, formulations are presented as below:

Mason interest $=\frac{\sum_{i} p_{i} \Delta_{i}}{\Delta}$

Here, $p_{i}$ is the forward path in signal diagram from input to the output, $\Delta_{i}$ represents the remaining path after traversing the forward path and $\Delta$ is the path with removing of the even loops and adding the odd loops. Therefore:

$$
\begin{aligned}
& p_{1}=\left(\alpha \% C^{1}\right)(1)\left(\frac{1}{D}\right)(c) \\
& \Delta_{1}=1 \\
& \Delta=1+\left[\alpha \% H(t) \frac{1}{D}\right] \\
& \text { Mason interest }=\frac{\alpha \% C^{1} \times \frac{1}{D}}{1+\alpha \% \frac{H(t)}{D}}
\end{aligned}
$$

The transformation function is as follows:

$G(s)=\frac{+\alpha \% \frac{1}{s} C^{\prime}}{1+\frac{1+(s)}{s}}=\frac{\alpha \% S^{-1} C^{\prime}}{1+S^{-1} H(s)} \times S^{2}=\frac{+\alpha \% S C^{1}}{S^{2}+H(s) S}$

Considering the equations below:

$$
\begin{gathered}
\frac{1}{D}=\frac{1}{S} \\
x(t)=x(s) \\
H(t)=H(s)
\end{gathered}
$$

The state equation is calculated as below:

$$
\begin{aligned}
a_{1} & =H(s) \\
a_{2} & =0 \\
a_{0} & =1 \\
b_{0} & =0 \\
b_{1} & =\alpha \% C^{1} \\
b_{2} & =0 \\
\beta_{0} & =0 \\
\beta_{1} & =b_{1}=\alpha \% C^{1} \\
\beta_{2} & =b_{2}-\alpha_{1} \beta_{1}=\left(0-H(s) \alpha \% C^{1}=-\alpha \% C H S\right) \\
\dot{\mathrm{x}}(t) & =\left[\begin{array}{cc}
0 & 1 \\
-H(t) & 0
\end{array}\right] x(t)+\left[\begin{array}{c}
+\alpha \% \\
-\alpha \% H(s)
\end{array}\right] u
\end{aligned}
$$

With regards to the stable state equation, the stability of the problem is investigated:

row $1 \begin{array}{cc}a_{0} & a_{2} \\ 1 & 0\end{array}$ 
row $3 b_{1}=0$

$b_{1}=\frac{a_{1} a_{2}-a_{0} a_{3}}{a_{1}}$

$b_{1}=0-0=0$

If decadence has been omitted unintentionally, the second solution would be:

$$
S^{2}+H(s) S \stackrel{s=\frac{1}{p}}{\Longrightarrow} \frac{1}{p^{2}}+\frac{H s}{p}=0 \stackrel{x p^{2}}{\Longrightarrow} 1+P H(s)=0
$$

1 row $p \quad 0$

2 row 10

3 row 0

According the obtained results, the system is stable in both states.

\section{Appendix 2. Determining the probability of the scenario occurrence}

For further explanation, formulations and computations are presented as following:

How to calculate $\mathrm{P}_{\mathrm{i}}$ (ith probability): First, some definitions are described in Table 10.

\section{Table 10}

Definition of parameters and variables

\begin{tabular}{cl}
\hline $\boldsymbol{x}_{\boldsymbol{i}}$ & Decision variable for player $\mathrm{i}$ \\
$\mathrm{f}_{\mathrm{i}}(\mathrm{x})$ & Objective function for player $\mathrm{i}$ \\
$g_{\mathrm{i}}(x)$ & Constraint for player $\mathrm{i}$ \\
$\lambda_{\mathrm{i}}$ & Lagrangian coefficient \\
$\mathrm{b}_{\mathrm{i}}$ & Source of player i \\
$\mathrm{B}_{\mathrm{i}}$ & Right hand coefficient of player $\mathrm{i}$ \\
$\mathrm{R}_{\mathrm{i}}(\mathrm{x})$ & Revenue \\
$\mathrm{P}_{\mathrm{i}}$ & Occurrence probabilities for player $\mathrm{i}$ \\
$\delta_{\mathrm{i}}$ & Matrix 1 \\
$\mathrm{x}_{\mathrm{j}}^{\mathrm{i}} \in x_{i}, \mathrm{j}=1,2$ & Free variable \\
$a_{j j}^{i}$ & Strategy $\mathrm{j}$ for player $\mathrm{i}$ \\
\hline
\end{tabular}

\section{A) Mathematical programming model for variable decision maker i:}

$\operatorname{Max} f(x)$

St $\quad g_{\mathrm{i}}(x) \geq 0$

$x_{i} \geq 0$

\section{B) Kuhn-Tucker (KKT) Conditions (I):}

By applying KKT conditions, model can changes as below:

$$
\begin{aligned}
& \operatorname{Min} \sum_{\mathrm{i}} \lambda_{\mathrm{i}} g_{\mathrm{i}}(\mathrm{x}) \\
& \nabla f_{\mathrm{i}}(\mathrm{x})+\sum \lambda_{\mathrm{i}} g_{\mathrm{i}}(\mathrm{x})=0 \quad \forall \mathrm{i} \\
& g_{\mathrm{i}}(\mathrm{x}) \geq 0
\end{aligned}
$$




$$
\lambda_{\mathrm{i}} \geq 0
$$

Assume that:

$g_{i}(x): B_{i} x \leq b_{i}$

So, we have: $\quad \nabla g_{i}(x)=-B_{i}$

On the other hand, We consider: $f_{\mathrm{i}}(\mathrm{x})=R \times \mathrm{x}=\mathrm{R}_{\mathrm{i}}(\mathrm{x}) \mathrm{x}$

Therefore, $\nabla f_{\mathrm{i}}(\mathrm{x})=\mathrm{R}_{\mathrm{i}}(\mathrm{x})$

$$
\operatorname{Min} \sum_{i} \lambda_{i}\left(b_{i}-B_{i} x\right)
$$

$$
\text { St. } R_{i}(x)-\sum \lambda_{i} B_{i}=0
$$

$$
\begin{aligned}
& b_{i}-B_{i}(x) \geq 0 \\
& \lambda_{i} \geq 0
\end{aligned}
$$

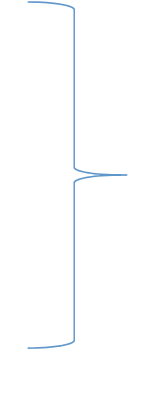

With respect to (III), we have for (II):

\begin{tabular}{|c|c|}
\hline & Second Player \\
\hline & $x_{1}^{2} \quad x_{2}^{2}$ \\
\hline First Player & 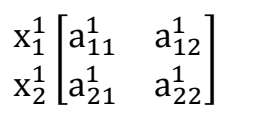 \\
\hline
\end{tabular}

$$
\begin{aligned}
& \mathrm{R}_{\mathrm{i}}(\mathrm{x})=\sum \lambda_{\mathrm{i}} \mathrm{B}_{\mathrm{i}} \\
& \rightarrow \underbrace{\mathrm{R}_{\mathrm{i}}(\mathrm{x}) x_{i}}_{\sum_{\mathrm{i}} \mathrm{f}(\mathrm{x})}=\sum_{\mathrm{i}} \lambda_{\mathrm{i}} \mathrm{B}_{\mathrm{i}} x_{\boldsymbol{i}}
\end{aligned}
$$

After rewriting (IV):

$$
\begin{gathered}
\operatorname{Min} \sum_{i} \lambda_{i} b_{i}-\sum_{i} f_{i}(x) \\
R_{i}(x)-\sum_{i} \lambda_{i} B_{i}=0 \\
b_{i}-B_{i}(x) \geq 0 \\
\lambda_{i} \geq 0
\end{gathered}
$$

$\mathrm{R}_{\mathrm{i}}(\mathrm{x})=:\left(\mathrm{x}_{1}^{1} \mathrm{x}_{2}^{1}\right)\left(\begin{array}{ll}\mathrm{a}_{11}^{1} & \mathrm{a}_{12}^{1} \\ \mathrm{a}_{21}^{1} & \mathrm{a}_{22}^{1}\end{array}\right)$

Desirabilities : $\left\{\begin{array}{l}a_{11}^{1} x_{1}^{1}+a_{21}^{1} x_{2}^{1} \\ a_{12}^{1} x_{1}^{1}+a_{22}^{1} x_{2}^{1}\end{array}\right.$

By considering second player in above equation:

$$
f_{1}(x)=\left(x_{1}^{1} x_{2}^{1}\right) \cdot\left(\begin{array}{ll}
a_{11}^{1} & a_{12}^{1} \\
a_{21}^{1} & a_{22}^{1}
\end{array}\right) \cdot\left(\begin{array}{l}
x_{1}^{2} \\
x_{2}^{2}
\end{array}\right)
$$

And objective for second player:
Analyze the problem with game condition:

$$
\mathrm{x}_{1}^{1} \text { : strategy } 1 \text { from player } 1
$$

:profit gained by game for player $1 \mathrm{a}_{11}^{1}$ using strategy 1 from player 1 , and strategy 1 from player 2 
$f_{2}(x)=\left(x_{1}^{1} x_{2}^{1}\right) \cdot\left(\begin{array}{ll}a_{11}^{2} & a_{12}^{2} \\ a_{21}^{2} & a_{22}^{2}\end{array}\right) \cdot\left(\begin{array}{l}x_{1}^{2} \\ x_{2}^{2}\end{array}\right)$

Then:

$\sum_{i=1}^{2} f_{i}(x)=\left(x_{1}^{1} x_{2}^{1}\right)\left[\left(\begin{array}{ll}a_{11}^{1} & a_{12}^{1} \\ a_{21}^{1} & a_{22}^{1}\end{array}\right)+\left(\begin{array}{ll}a_{11}^{2} & a_{12}^{2} \\ a_{21}^{2} & a_{22}^{2}\end{array}\right) \cdot\left(\begin{array}{l}x_{1}^{2} \\ x_{2}^{2}\end{array}\right)\right]$

Now, we analyze randomness condition of game; according to occurrence probability for each player in above model:

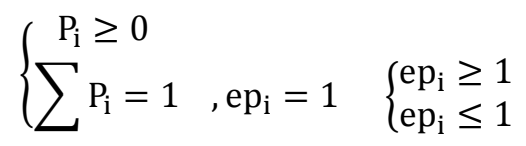

$\rightarrow\left\{\begin{array}{l}\left.(1) \mathrm{P}_{\mathrm{i}} \geq 0 \quad \overline{\mathrm{S}}_{\mathrm{i}}\right) \\ \text { (2) } \mathrm{ep}_{\mathrm{i}}-1 \geq 0\left(\beta_{\mathrm{i}}\right) \\ (3) 1-\mathrm{ep}_{\mathrm{i}} \geq 0\left(\alpha_{\mathrm{i}}\right)\end{array}\right.$

according to KKT condition $\rightarrow \mathrm{b}_{\mathrm{i}}-\mathrm{B}_{\mathrm{i}}\left(\mathrm{x}_{\mathrm{i}}\right) \geq 0$

$\rightarrow \mathrm{b}_{\mathrm{i}}=\left(\begin{array}{c}0 \\ +1 \\ -1\end{array}\right), \quad \mathrm{B}_{\mathrm{i}}=\left(\begin{array}{c}-\mathrm{I} \\ +\mathrm{e} \\ -\mathrm{e}\end{array}\right), \boldsymbol{x}_{\boldsymbol{i}}=\mathrm{p}_{\mathrm{i}}$

,e $=(1,1, \ldots),-\mathrm{e}(-1, \ldots)$

,$\lambda_{\mathrm{i}}\left(\begin{array}{c}s_{\mathrm{i}} \\ \alpha_{\mathrm{i}} \\ \beta_{\mathrm{i}}\end{array}\right)$

By replacing above formulations in Eq. ( $\underline{\nabla})$ :

$$
\begin{aligned}
& \operatorname{Max} \sum E \mathrm{f}_{\mathrm{i}}(\mathrm{x})-\sum \delta_{\mathrm{i}} \\
& \mathrm{R}_{\mathrm{i}}\left(\mathrm{P}_{\mathrm{i}}\right)-\delta_{\mathrm{i}} \mathrm{e} \leq 0 \\
& \mathrm{P}_{\mathrm{i}} \geq 0 \\
& \mathrm{ep}_{\mathrm{i}}=1
\end{aligned}
$$

$\delta_{\mathrm{i}}=$ free variable

In the presented model, among all three sections of the city, the first and the third section are competing with each other and their game tables are shown based on the two criteria of traffic and population density in below. The second section, which is a transformation place, is concluded from the other two sections. The first and second row (p1 and p2) are obtained from the first criterion:

$$
\left[\begin{array}{cc}
2 & -1 \\
-1 & 1
\end{array}\right]
$$

For $\mathrm{p} 3$ and $\mathrm{p} 4$ which are obtained from the second criterion, we have:

$$
\left[\begin{array}{cc}
1 & -1 \\
-1 & 2
\end{array}\right]
$$

Thus, using the mathematical model, values for $\mathrm{p}$ are calculated as below (Wang et al., 2016):

$$
\operatorname{Max}(p 1, p 2)\left[\begin{array}{cc}
2+1 & -1-1 \\
-1-1 & 1+2
\end{array}\right]\left(\begin{array}{l}
p 3 \\
p 4
\end{array}\right)-(\rho 1+\rho 2)
$$


St .

$$
\begin{aligned}
& (p 1, p 2)\left[\begin{array}{cc}
2 & -1 \\
-1 & 1
\end{array}\right]-(\rho 1+\rho 2) \leq 0 \\
& (p 3, p 4)\left[\begin{array}{cc}
1 & -1 \\
-1 & 2
\end{array}\right]-(\rho 1+\rho 2) \leq 0 \\
& p 1+p 2=1 \\
& p 3+p 4=1 \\
& p 1, p 2, p 3, p 4, \rho 1, \rho 2 \geq 0
\end{aligned}
$$

where $\rho 1$ and $\rho 2$ are the Lagrange coefficients for each criteria and p5 (which is shown in Table 6) is proportional to the sum of them.

\section{References}

Aly, A. A., \& White, J. A. (1978). Probabilistic formulation of the emergency service location problem. Journal of the Operational Research Society, 29(12), 1167-1179.

Araghi, S.M.., Tavallaei, S., Ziaeian, P. (2011). Location analysis regarding disaster management bases via GIS, Case study: Tehran Municipality (No.6). Urban - Regional Studies and Research Journal, 3(10).

Bahadori, M., Nasiripur, A., Tofighi, S., \& Gohari, M. (2010). Emergency Medical Services In Iran: An Overview. Australasian Medical Journal, 3(6).

Berlin, G. N., \& Liebman, J. C. (1974). Mathematical analysis of emergency ambulance location. Socio-Economic Planning Sciences, 8(6), 323-328.

Balcik, B., \& Beamon, B. M. (2008). Facility location in humanitarian relief. International Journal of Logistics, 11(2), 101-121.

Ball, M. O., \& Lin, F. L. (1993). A reliability model applied to emergency service vehicle location. Operations research, 41(1), 18-36.

Campbell, A. M., Vandenbussche, D., \& Hermann, W. (2008). Routing for relief efforts. Transportation Science, 42(2), 127-145.

Campos, V., Bandeira, R., \& Bandeira, A. (2012). A method for evacuation route planning in disaster situations. Procedia-Social and Behavioral Sciences, 54, 503-512.

Charnes, A., Cooper, W. W., \& Rhodes, E. (1978). Measuring the efficiency of decision making units. European journal of operational research, 2(6), 429-444.

Cooper, W. W., Seiford, L. M., \& Zhu, J. (2004). Data envelopment analysis. In Handbook on data envelopment analysis (pp. 1-39). Springer US.

Daskin, M. S. (1983). A maximum expected covering location model: formulation, properties and heuristic solution. Transportation Science, 17(1), 48-70.

Davis, L. B., Samanlioglu, F., Qu, X., \& Root, S. (2013). Inventory planning and coordination in disaster relief efforts. International Journal of Production Economics, 141(2), 561-573.

Edrissi, A., Poorzahedy, H., Nassiri, H., \& Nourinejad, M. (2013). A multi-agent optimization formulation of earthquake disaster prevention and management. European Journal of Operational Research, 229(1), 261-275.

Forrester, A. T. (1961). Photoelectric mixing as a spectroscopic tool. JOSA, 51(3), 253-256.

Hwang, C. R. (1988). Simulated annealing: theory and applications. Acta Applicandae Mathematicae, 12(1), 108-111.

Jotshi, A., Gong, Q., \& Batta, R. (2009). Dispatching and routing of emergency vehicles in disaster mitigation using data fusion. Socio-Economic Planning Sciences, 43(1), 1-24.

Karasakal, O., \& Karasakal, E. K. (2004). A maximal covering location model in the presence of partial coverage. Computers \& Operations Research, 31(9), 1515-1526.

Liberatore, F., Ortuño, M. T., Tirado, G., Vitoriano, B., \& Scaparra, M. P. (2014). A hierarchical compromise model for the joint optimization of recovery operations and distribution of emergency goods in Humanitarian Logistics. Computers \& Operations Research, 42, 3-13. 
Matsutomi, T., \& Ishii, H. (1992, March). An emergency service facility location problem with fuzzy objective and constraint. In Fuzzy Systems, 1992., IEEE International Conference on (pp. 315-322). IEEE.

Mavrotas, G., \& Trifillis, P. (2006). Multicriteria decision analysis with minimum information: combining DEA with MAVT. Computers \& operations research, 33(8), 2083-2098.

Mete, H. O., \& Zabinsky, Z. B. (2007). Preparing for disasters: medical supply location and distribution. In Proceedings of the INFORMS conference, Seattle, WA.

Ogata, K. (2010). Modern Control Engineering. $5^{\text {th }}$ ed., Prentice Hall.

Overstreet, R. E., Hall, D., Hanna, J. B., \& Kelly Rainer Jr, R. (2011). Research in humanitarian logistics. Journal of Humanitarian Logistics and Supply Chain Management, 1(2), 114-131.

Özdamar, L., Ekinci, E., \& Küçükyazici, B. (2004). Emergency logistics planning in natural disasters. Annals of operations research, 129(1-4), 217-245.

Pirkul, H., \& Schilling, D. A. (1988). The siting of emergency service facilities with workload capacities and backup service. Management Science, 34(7), 896-908.

Ratick, S., Meacham, B., \& Aoyama, Y. (2008). Locating backup facilities to enhance supply chain disaster resilience. Growth and Change, 39(4), 642-666.

ReVelle, C. (1989). Review, extension and prediction in emergency service siting models. European Journal of Operational Research, 40(1), 58-69.

Rahmaniani, R., \& Shafia, M. A. (2013). A study on maximum covering transportation network design with facility location under uncertainty. Journal of Industrial and Production Engineering, 30(2), 78-93.

Shen, Z. J. M., Pannala, J., Rai, R., \& Tsoi, T. S. (2008). Modeling transportation networks during disruptions and emergency evacuations. University of California Transportation Center.

Swoveland, C., Uyeno, D., Vertinsky, I., \& Vickson, R. (1973). Ambulance location: a probabilistic enumeration approach. Management Science, 20(4-part-ii), 686-698.

Tzeng, G. H., Cheng, H. J., \& Huang, T. D. (2007). Multi-objective optimal planning for designing relief delivery systems. Transportation Research Part E: Logistics and Transportation Review, 43(6), 673-686.

Wang, L., Zhou, Z., \& Wu, W. (2016). Game theory-based model for maximizing SSP utility in cognitive radio networks. Computer Communications, 86, 29-39.

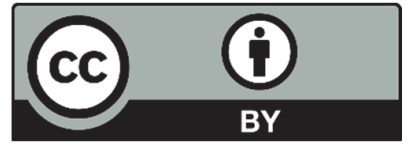

(C) 2016 by the authors; licensee Growing Science, Canada. This is an open access article distributed under the terms and conditions of the Creative Commons Attribution (CC-BY) license (http://creativecommons.org/licenses/by/4.0/). 